\title{
A congruence involving harmonic sums modulo $p^{\alpha} q^{\beta}$
}

\author{
TianXin CAI ${ }^{1 *}$ Zhongyan Shen $^{2 * *}$ LiRui JiA ${ }^{1 * * *}$ \\ 1 Department of Mathematics, Zhejiang University \\ Hangzhou 310027, P.R. China \\ 2 Department of Mathematics, Zhejiang International Study University \\ Hangzhou 310012, P.R. China \\ *txcai@zju.edu.cn \\ **huanchenszyan@163.com \\ $* * *$ jialirui@126.com
}

\begin{abstract}
In 2014, Wang and Cai established the following harmonic congruence for any odd prime $p$ and positive integer $r$,

$$
Z\left(p^{r}\right) \equiv-2 p^{r-1} B_{p-3}\left(\bmod p^{r}\right),
$$
\end{abstract}

where $Z(n)=\sum_{\substack{i+j+k=n \\ i, j, k \in \mathcal{P}_{n}}} \frac{1}{i j k}$ and $\mathcal{P}_{n}$ denote the set of positive integers which are prime to $n$.

In this note, we obtain a congruence for distinct odd primes $p, q$ and positive integers $\alpha, \beta$,

$$
Z\left(p^{\alpha} q^{\beta}\right) \equiv 2(2-q)\left(1-\frac{1}{q^{3}}\right) p^{\alpha-1} q^{\beta-1} B_{p-3} \quad\left(\bmod p^{\alpha}\right)
$$

and the necessary and sufficient condition for

$$
Z\left(p^{\alpha} q^{\beta}\right) \equiv 0 \quad\left(\bmod p^{\alpha} q^{\beta}\right) .
$$

Finally, we raise a conjecture that for $n>1$ and odd prime power $p^{\alpha}|| n, \alpha \geq 1$,

$$
Z(n) \equiv \prod_{\substack{q \mid n \\ q \neq p}}\left(1-\frac{2}{q}\right)\left(1-\frac{1}{q^{3}}\right)\left(-\frac{2 n}{p}\right) B_{p-3} \quad\left(\bmod p^{\alpha}\right) .
$$

Keywords Bernoulli numbers, harmonic sums, congruences MSC 11A07, 11A41

This work is supported by the National Natural Science Foundation of China, Project (No.10871169) and the Natural Science Foundation of Zhejiang Province, Project (No. LQ13A010012). 


\section{Introduction.}

Let

$$
Z(n)=\sum_{\substack{i+j+k=n \\ i, j, k \in \mathcal{P}_{n}}} \frac{1}{i j k},
$$

where $\mathcal{P}_{n}$ denotes the set of positive integers which are prime to $n$.

At the beginning of the 21th century, Zhao (Cf.[10]) first announced the following curious congruence involving multiple harmonic sums for any odd prime $p>3$,

$$
Z(p) \equiv-2 B_{p-3}(\bmod p),
$$

which holds when $p=3$ evidently. Here, Bernoulli numbers $B_{k}$ are defined by the recursive relation:

$$
\sum_{i=0}^{n}\left(\begin{array}{c}
n+1 \\
i
\end{array}\right) B_{i}=0, n \geq 1 .
$$

A simple proof of (1) was presented in [3. This congruence has been generalized along several directions. First, Zhou and Cai [11] established the following harmonic congruence for prime $p>3$ and integer $n \leq p-2$

$$
\sum_{l_{1}+l_{2}+\cdots+l_{n}=p} \frac{1}{l_{1} l_{2} \cdots l_{n}} \equiv\left\{\begin{array}{l}
-(n-1) ! B_{p-n}(\bmod p) \quad \text { if } 2 \nmid n, \\
-\frac{n(n !)}{2(n+1)} p B_{p-n-1}\left(\bmod p^{2}\right) \quad \text { if } 2 \mid n .
\end{array}\right.
$$

Later, Xia and Cai $[8$ generalized (11) to

$$
Z(p) \equiv-\frac{12 B_{p-3}}{p-3}-\frac{3 B_{2 p-4}}{p-4}\left(\bmod p^{2}\right),
$$

where $p>5$ is a prime.

Recently, Wang and Cai [7] proved for every prime $p \geq 3$ and positive integer $r$,

$$
Z\left(p^{r}\right) \equiv-2 p^{r-1} B_{p-3}\left(\bmod p^{r}\right) .
$$

Let $n=2$ or 4 , for every positive integer $r \geq \frac{n}{2}$ and prime $p>n$, Zhao [9] generalized (2) to

$$
\sum_{\substack{i_{1}+i_{2}+\cdots+i_{n}=p^{r} \\ i_{1}, i_{2}, \cdots, i_{n} \in \mathcal{P}_{p}}} \frac{1}{i_{1} i_{2} \cdots i_{n}} \equiv-\frac{n !}{n+1} p^{r} B_{p-n-1}\left(\bmod p^{r+1}\right) .
$$

In this paper, we obtain the following theorems.

Theorem 1. Let $p, q$ be distinct odd primes, then

$$
Z(p q) \equiv 2(2-q)\left(1-\frac{1}{q^{3}}\right) B_{p-3} \quad(\bmod p) .
$$


Theorem 2. Let $p, q$ be distinct odd primes and $\alpha, \beta$ positive integers, then

$$
Z\left(p^{\alpha} q^{\beta}\right) \equiv 2(2-q)\left(1-\frac{1}{q^{3}}\right) p^{\alpha-1} q^{\beta-1} B_{p-3} \quad\left(\bmod p^{\alpha}\right) .
$$

Theorem 3. Let $p, q$ be distinct odd primes and $\alpha, \beta$ positive integers, if and only if $p=q^{2}+q+1$ or $q=p^{2}+p+1$ or $p \mid q^{2}+q+1$ and $q \mid p^{2}+p+1$, we have

$$
Z\left(p^{\alpha} q^{\beta}\right) \equiv 0 \quad\left(\bmod p^{\alpha} q^{\beta}\right) .
$$

Finally, we have the following

Conjecture For any positive integer $n>1$ and odd prime power $p^{\alpha}|| n\left(p^{\alpha} \mid n\right.$, $\left.p^{\alpha+1} \nmid n\right), \alpha \geq 1$, then

$$
Z(n) \equiv \prod_{\substack{q \mid n \\ q \neq p}}\left(1-\frac{2}{q}\right)\left(1-\frac{1}{q^{3}}\right)\left(-\frac{2 n}{p}\right) B_{p-3} \quad\left(\bmod p^{\alpha}\right) .
$$

This is the generalization of Theorem 2 and (2).

\section{Preliminaries.}

In order to prove the theorems, we need the following lemmas.

Lemma 1 ([7]). Let $p$ be odd prime and $r, m$ positive integers, then

$$
\begin{gathered}
Z\left(p^{r}\right) \equiv-2 p^{r-1} B_{p-3} \quad\left(\bmod p^{r}\right), \\
\sum_{\substack{i+j+k=m p^{r} \\
i, j, k \in \mathcal{P}_{p}}} \frac{1}{i j k} \equiv m Z\left(p^{r}\right) \quad\left(\bmod p^{r}\right) .
\end{gathered}
$$

Lemma $2([4,[6])$. Let $p$ be odd prime and $l$ positive integer, then

$$
\sum_{\substack{k=1 \\
(k, p)=1}}^{p^{l}-1} \frac{1}{k^{s}} \equiv\left\{\begin{array}{l}
0\left(\bmod p^{2 l-1}\right), \text { for odd } s \text { with } p-1 \mid s+1 \text { and } p \nmid s, \\
0\left(\bmod p^{2 l}\right), \text { for odd } s \text { with } p-1 \nmid s+1 \text { or } p \mid s \\
0\left(\bmod p^{l-1}\right), \text { for even } s \text { with } p-1 \mid s \\
0\left(\bmod p^{l}\right), \text { for even } s \text { with } p-1 \nmid s .
\end{array}\right.
$$

Define

$$
S(n ; p)=\sum_{\substack{a=1 \\(a, p)=1}}^{n-1} \frac{1}{a^{2}} \sum_{\substack{i=1 \\(i, p)=1}}^{a m-1} \frac{1}{i}, T(n ; p)=\sum_{\substack{a=1 \\(a, p)=1}}^{n-1} \frac{1}{a^{2}} \sum_{\substack{i=1 \\ i \equiv a m}}^{a m-1} \frac{1}{i}
$$

Lemma 3. Let $p$ be odd prime and $m$ positive integer coprime to $p$, then

$$
S(p ; p) \equiv m^{2} B_{p-3} \quad(\bmod p) .
$$


Proof. When $(i, p)=1$, then $\frac{1}{i} \equiv i^{p-2}(\bmod p)$ by Euler's Theorem. For any positive integers $n$ and $r$, it is well-known that

$$
\sum_{a=1}^{n-1} a^{r}=\frac{1}{r+1} \sum_{k=0}^{r}\left(\begin{array}{c}
r+1 \\
k
\end{array}\right) B_{k} n^{r+1-k}
$$

hence

$$
\begin{aligned}
\sum_{a=1}^{p-1} \frac{1}{a^{2}} \sum_{\substack{i=1 \\
(i, p)=1}}^{a m-1} \frac{1}{i} & \equiv \sum_{a=1}^{p-1} \frac{1}{a^{2}} \sum_{\substack{i=1 \\
(i, p)=1}}^{a m-1} i^{p-2} \equiv \sum_{a=1}^{p-1} \frac{1}{a^{2}} \sum_{i=1}^{a m-1} i^{p-2} \\
& \equiv \sum_{a=1}^{p-1} \frac{1}{a^{2}} \frac{1}{p-1} \sum_{k=0}^{p-2}\left(\begin{array}{c}
p-1 \\
k
\end{array}\right) B_{k}(a m)^{p-1-k} \\
& \equiv \frac{m^{2}}{p-1} \sum_{k=0}^{p-2} \frac{1}{p-1}\left(\begin{array}{c}
p-1 \\
k
\end{array}\right) B_{k} \sum_{a=1}^{p-1}(a m)^{p-3-k} \quad(\bmod p) .(4)
\end{aligned}
$$

Since $(a m, p)=1$, by Lemma 2 , if and only if $k=p-3, \sum_{a=1}^{p-1}(a m)^{p-3-k}$ is not congruence to 0 modulo $p$. It follows from (4) that

$$
\sum_{a=1}^{p-1} \frac{1}{a^{2}} \sum_{\substack{i=1 \\
(i, p)=1}}^{a m-1} \frac{1}{i} \equiv \frac{m^{2}}{p-1}\left(\begin{array}{c}
p-1 \\
p-3
\end{array}\right) B_{p-3}(p-1) \equiv m^{2} B_{p-3} \quad(\bmod p) .
$$

This completes the proof of Lemma 3.

Lemma 4. Let $p$ be odd prime, $m$ positive integer coprime to $p$ and $\alpha \geq 2$ positive integer, then

$$
S\left(p^{\alpha} ; p\right) \equiv p^{\alpha-1} m^{2} B_{p-3} \quad\left(\bmod p^{\alpha}\right) .
$$

Proof. Let $a=s+p^{\alpha-1} t, 1 \leq s \leq p^{\alpha-1}-1,0 \leq t \leq p-1,(s, p)=1$, then

$$
\begin{aligned}
S\left(p^{\alpha} ; p\right) & =\sum_{t=0}^{p-1} \sum_{\substack{s=1 \\
(s, p)=1}}^{p^{\alpha-1}-1} \frac{1}{\left(s+p^{\alpha-1} t\right)^{2}} \sum_{\substack{i=1 \\
(i, p)=1}}^{\left(s+p^{\alpha-1} t\right) m-1} \frac{1}{i} \\
& \equiv \sum_{t=0}^{p-1} \sum_{\substack{s=1 \\
p^{\alpha-1}-1}} \frac{1}{s^{2}}\left(1-\frac{2 p^{\alpha-1} t}{s}\right) \sum_{\substack{i=1 \\
s m-p)=1 \\
(i, p)=1}}^{s m-1} \frac{1}{i} \\
& +\sum_{t=0}^{p-1} \sum_{\substack{s=1 \\
(s, p)=1}}^{p^{\alpha-1}-1} \frac{1}{s^{2}}\left(1-\frac{2 p^{\alpha-1} t}{s}\right) \sum_{\substack{i=s m \\
(i, p)=1}}^{s m+p^{\alpha-1} t m-1} \frac{1}{i}\left(\bmod p^{\alpha}\right) .
\end{aligned}
$$

It is easy to see that

$$
2 p^{\alpha-1} \sum_{t=0}^{p-1} t \sum_{\substack{s=1 \\(s, p)=1}}^{p^{\alpha-1}-1} \frac{1}{s^{3}} \sum_{\substack{i=1 \\(i, p)=1}}^{s m-1} \frac{1}{i} \equiv 0 \quad\left(\bmod p^{\alpha}\right) .
$$


By Lemma 2, we have

$$
\sum_{t=0}^{p-1} \sum_{\substack{s=1 \\(s, p)=1}}^{p^{\alpha-1}-1} \frac{1}{s^{2}}\left(1-\frac{2 p^{\alpha-1} t}{s}\right) \sum_{\substack{i=s m \\(i, p)=1}}^{s m+p^{\alpha-1} t m-1} \frac{1}{i} \equiv 0 \quad\left(\bmod p^{\alpha}\right) .
$$

Therefore

$S\left(p^{\alpha} ; p\right) \equiv \sum_{t=0}^{p-1} \sum_{\substack{s=1 \\(s, p)=1}}^{p^{\alpha-1}-1} \frac{1}{s^{2}} \sum_{\substack{i=1 \\(i, p)=1}}^{s m-1} \frac{1}{i} \equiv p S\left(p^{\alpha-1} ; p\right) \equiv \cdots \equiv p^{\alpha-1} S(p ; p) \quad\left(\bmod p^{\alpha}\right)$.

By Lemma 3, we complete the proof of Lemma 4.

Lemma 5. Let $p, q$ be distinct odd primes, $m$ positive integer coprime to $p$ and $\alpha \geq 2, \beta \geq 0$ integers, then

$$
S\left(p^{\alpha} q^{\beta} p\right) \equiv p^{\alpha-1} q^{\beta} m^{2} B_{p-3} \quad\left(\bmod p^{\alpha}\right) .
$$

Proof. Let $a=s+p^{\alpha} t, 1 \leq s \leq p^{\alpha}-1,0 \leq t \leq q^{\beta}-1,(s, p)=1$, then

$$
\begin{aligned}
S\left(p^{\alpha} q^{\beta} ; p\right) & =\sum_{t=0}^{q^{\beta}-1} \sum_{\substack{s=1 \\
(s, p)=1}}^{p^{\alpha}-1} \frac{1}{\left(s+p^{\alpha} t\right)^{2}} \sum_{\substack{i=1 \\
(i, p)=1}}^{\left(s+p^{\alpha} t\right) m-1} \frac{1}{i} \\
& \equiv \sum_{t=0}^{q^{\beta}-1} \sum_{\substack{s=1 \\
p^{\alpha}-1}} \frac{1}{s^{2}} \sum_{\substack{i=1 \\
(s, p)=1}}^{s m-1} \frac{1}{i} \\
& +\sum_{t=0}^{q^{\beta}-1} \sum_{\substack{s=1 \\
(s, p)=1}}^{p^{\alpha}-1} \frac{1}{s^{2}} \sum_{\substack{i=s m \\
(i, p)=1}}^{s m+p^{\alpha} t m-1} \frac{1}{i}\left(\bmod p^{\alpha}\right) .
\end{aligned}
$$

By Lemma 2, we have

$$
\sum_{\substack{i=s m \\(i, p)=1}}^{s m+p^{\alpha} t m-1} \frac{1}{i} \equiv 0 \quad\left(\bmod p^{\alpha}\right)
$$

Therefore

$$
S\left(p^{\alpha} q^{\beta} ; p\right) \equiv q^{\beta} S\left(p^{\alpha}\right) \quad\left(\bmod p^{\alpha}\right) .
$$

By Lemma 4, we complete the proof of Lemma 5.

Lemma 6 ([5]). Let $p$ be odd prime, $m \in Z^{+},(m, p)=1$, $[x]$ denote the largest integer less than or equal to $x$, then

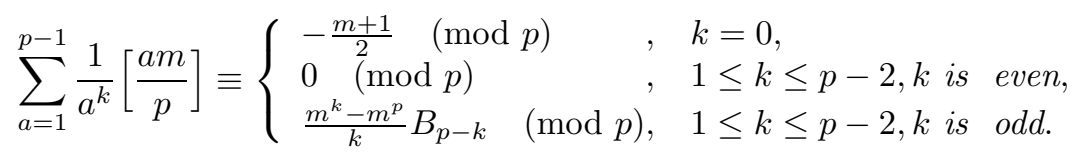


Meanwhile, it is easy to see that

$$
T(p ; p) \equiv \frac{1}{m} \sum_{a=1}^{p-1} \frac{1}{a^{3}}\left[\frac{a m}{p}\right] \equiv \frac{m^{3}-m}{3 m} B_{p-3} \quad(\bmod p) .
$$

Lemma 7. Let $p$ be odd prime, $m$ positive integer coprime to $p$ and $\alpha \geq 2$ integer, then

$$
T\left(p^{\alpha} ; p\right) \equiv \frac{m^{3}-m}{3 m} p^{\alpha-1} B_{p-3} \quad\left(\bmod p^{\alpha}\right) .
$$

Proof. Let $a=s+p^{\alpha-1} t, 1 \leq s \leq p^{\alpha-1}-1,0 \leq t \leq p-1,(s, p)=1$, then

$$
\begin{aligned}
T\left(p^{\alpha} ; p\right) & =\sum_{\substack{a=1 \\
(a, p)=1}}^{p^{\alpha}-1} \frac{1}{a^{2}} \sum_{\substack{i=1 \\
i \equiv a m}}^{a m-1} \frac{1}{i}=\sum_{t=0}^{p-1} \sum_{\substack{s=1 \\
(s, p)=1}}^{p^{\alpha-1}-1} \frac{1}{\left(s+p^{\alpha-1} t\right)^{2}} \sum_{\substack{i=1 \\
i \equiv\left(s+p^{\alpha-1} t\right) m}}^{\left(s+p^{\alpha-1} t\right) m-1} \frac{1}{i}(\bmod p) \\
& \equiv \sum_{t=0}^{p-1} \sum_{\substack{s=1 \\
(s, p)=1}}^{p^{\alpha-1}-1} \frac{1}{s^{2}}\left(1-\frac{2 p^{\alpha-1} t}{s}\right)\left(\sum_{\substack{i=1 \\
i \equiv s m}}^{s m-1} \frac{1}{i}+\sum_{\substack{i=s m \\
i \equiv s m}}^{s m+p^{\alpha-1} t m-1} \frac{1}{i}\right)(\bmod p)
\end{aligned}
$$

It is easy to see that

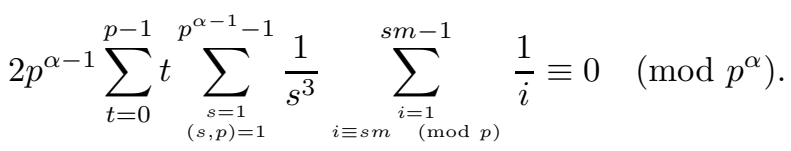

Since

$$
\begin{aligned}
\sum_{\substack{i=s m \\
i \equiv s m}}^{s m+p^{\alpha-1} t m-1} \frac{1}{i} & \equiv \sum_{j=0}^{p^{\alpha-2} t m-1} \frac{1}{s m+j p} \equiv \sum_{j=0}^{p^{\alpha-2} t m-1} \frac{1}{s m\left(1+\frac{j p}{s m}\right)} \equiv \sum_{j=0}^{p^{\alpha-2} t m-1} \frac{1}{s m} \sum_{k=0}^{\alpha-1}\left(-\frac{j p}{s m}\right)^{k} \\
& \equiv \sum_{k=0}^{\alpha-1} \frac{(-p)^{k}}{(s m)^{k+1}} \sum_{j=0}^{p^{\alpha-2} t m-1} j^{k}
\end{aligned}
$$

by (3), we have $\sum_{j=0}^{p^{\alpha-2} t m-1} j^{k} \equiv 0\left(\bmod p^{\alpha-2}\right)$. Together with Lemma 2 , we have

$$
\sum_{t=0}^{p-1} \sum_{\substack{s=1 \\(s, p)=1}}^{p^{\alpha-1}-1} \frac{1}{s^{2}}\left(1-\frac{2 p^{\alpha-1} t}{s}\right) \sum_{\substack{i=s m \\ i \equiv s m}}^{s m+p_{\bmod p)}^{\alpha-1} t m-1} \frac{1}{i} \equiv 0 \quad\left(\bmod p^{\alpha}\right)
$$

Hence

$T\left(p^{\alpha} ; p\right) \equiv \sum_{t=0}^{p-1} \sum_{\substack{s=1 \\(s, p)=1}}^{p^{\alpha-1}-1} \frac{1}{s^{2}} \sum_{\substack{i=1 \\ i \equiv s m}}^{s m-1} \frac{1}{(\bmod p)} \in \equiv T\left(p^{\alpha-1} ; p\right) \equiv \cdots \equiv p^{\alpha-1} T(p ; p) \quad\left(\bmod p^{\alpha}\right)$,

By (5), we complete the proof of Lemma 7 . 
Lemma 8. Let $p, q$ be distinct odd primes, $m$ positive integer coprime to $p$ and $\alpha \geq 2, \beta \geq 0$ integers, then

$$
T\left(p^{\alpha} q^{\beta} ; p\right) \equiv \frac{m^{3}-m}{3 m} p^{\alpha-1} q^{\beta} B_{p-3} \quad\left(\bmod p^{\alpha}\right) .
$$

Proof. Let $a=s+p^{\alpha} t, 1 \leq s \leq p^{\alpha}-1,0 \leq t \leq q^{\beta-1}-1,(s, p)=1$, then

$$
\begin{aligned}
T\left(p^{\alpha} q^{\beta} ; p\right) & =\sum_{t=0}^{q^{\beta}-1} \sum_{\substack{s=1 \\
(s, p)=1}}^{p^{\alpha}-1} \frac{1}{\left(s+p^{\alpha} t\right)^{2}} \sum_{\substack{i=1 \\
i \equiv\left(s+p^{\alpha} t\right) m}}^{\left(s+p^{\alpha} t\right) m-1} \frac{1}{i}(\bmod p) \\
& \equiv \sum_{t=0}^{q^{\beta}-1} \sum_{\substack{s=1 \\
(s, p)=1}}^{p^{\alpha}-1} \frac{1}{s^{2}} \sum_{\substack{i \equiv s m \\
i=1 \\
(\bmod p)}}^{s m-1} \frac{1}{i}+\sum_{t=0}^{q^{\beta}-1} \sum_{\substack{s=1 \\
(s, p)=1}}^{p^{\alpha}-1} \frac{1}{s^{2}} \sum_{\substack{i=s m \\
i=s m}}^{s m+p^{\alpha} t m-1} \frac{1}{i} \quad\left(\bmod p^{\alpha}\right) .
\end{aligned}
$$

Since

$$
\sum_{\substack{i=s m \\ i \equiv s m,(\bmod p)}}^{s m+p^{\alpha} t m-1} \frac{1}{i} \equiv \sum_{j=0}^{p^{\alpha-1} t m-1} \frac{1}{s m+j p} \quad\left(\bmod p^{\alpha}\right)
$$

similar to Lemma 6, we can prove

$$
\sum_{t=0}^{q^{\beta}-1} \sum_{\substack{s=1 \\(s, p)=1}}^{p^{\alpha}-1} \sum_{\substack{i=s m \\ s^{2}}}^{s m+p^{\alpha} t m-1} \frac{1}{i} \equiv 0 \quad\left(\bmod p^{\alpha}\right)
$$

Therefore

$$
T\left(p^{\alpha} q^{\beta} ; p\right) \equiv \sum_{t=0}^{q^{\beta}-1} \sum_{\substack{s=1 \\(s, p)=1}}^{p^{\alpha}-1} \frac{1}{s^{2}} \sum_{\substack{i=1 \\ i \equiv s m}}^{s m-1} \frac{1}{(\bmod p)} \in q^{\beta} T\left(p^{\alpha} ; p\right) \quad\left(\bmod p^{\alpha}\right) .
$$

By Lemma 7, we complete the proof of Lemma 8.

\section{Proofs of the Theorems.}

Proof of Theorem 1. By symmetry, It is easy to see that

$$
Z(p q)=\sum_{\substack{i+j+k=p q \\ i, j, k \in \mathcal{P} p q}} \frac{1}{i j k}=\sum_{\substack{i+j+k=p q \\ i, j, k \in \mathcal{P}_{p}}} \frac{1}{i j k}-3 \sum_{a=1}^{p-1} \frac{1}{(p-a) q} \sum_{\substack{i+j=a q \\(i j, p)=1}} \frac{1}{i j}+2 \sum_{\substack{a+b+c=p \\ a, b, c \in \mathcal{P} p}} \frac{1}{a q b q c q} .
$$

By Lemma 1,

$$
\sum_{\substack{i+j+k=p q \\ i, j, k \in \mathcal{P}_{p}}} \frac{1}{i j k}+2 \sum_{\substack{a+b+c=p \\ a, b, c \in \mathcal{P}_{p}}} \frac{1}{a q b q c q} \equiv\left(q+\frac{2}{q^{3}}\right) Z(p) \equiv-2\left(q+\frac{2}{q^{3}}\right) B_{p-3} \quad(\bmod p) .
$$


Again by symmetry, the second sum in (6) equals to

$$
\begin{aligned}
& -3 \sum_{a=1}^{p-1} \frac{1}{(p-a) q} \frac{1}{a q} \sum_{\substack{i+j=a q \\
(i j, p)=1}} \frac{i+j}{i j} \\
\equiv & \frac{6}{q^{2}} \sum_{a=1}^{p-1} \frac{1}{a^{2}} \sum_{\substack{i=1 \\
(i, p)=(a q-i, p)=1}}^{a q} \frac{1}{i} \\
\equiv & \frac{6}{q^{2}} \sum_{a=1}^{p-1} \frac{1}{a^{2}} \sum_{\substack{i=1 \\
a q-1}}^{\substack{i, p)=1 \\
i}} \frac{6}{q^{2}} \sum_{a=1}^{p-1} \frac{1}{a^{2}} \frac{1}{a q}\left[\frac{a q}{p}\right] \\
\equiv & \frac{6}{q^{2}} \sum_{a=1}^{p-1} \frac{1}{a^{2}} \sum_{\substack{i=1 \\
(i, p)=1}} \frac{1}{i}-\frac{6}{q^{3}} \sum_{a=1}^{p-1} \frac{1}{a^{3}}\left[\frac{a q}{p}\right] \quad(\bmod p) .
\end{aligned}
$$

Applying Lemma 3 and Lemma 6 to (8), we have

$$
-3 \sum_{a=1}^{p-1} \frac{1}{(p-a) q} \sum_{\substack{i+j=a q \\(i j, p)=1}} \frac{1}{i j} \equiv \frac{6}{q^{2}} q^{2} B_{p-3}-\frac{6}{q^{3}} \frac{q^{3}-q}{3} B_{p-3} \equiv 2\left(1+\frac{1}{q^{2}}\right) B_{p-3} \quad(\bmod p) .
$$

Combining (6), (7) and (9), we have

$Z(p q) \equiv-2\left(q+\frac{2}{q^{3}}\right) B_{p-3}+2\left(1+\frac{1}{q^{2}}\right) B_{p-3} \equiv 2(2-q)\left(1-\frac{1}{q^{3}}\right) B_{p-3} \quad(\bmod p)$.

This completes the proof of Theorem 1.

Remark 1 When $n=p q, p, q$ are distinct odd primes,

$Z(n) \equiv 2(2-q)\left(1-\frac{1}{q^{3}}\right) B_{p-3} \equiv 6\left(1+\frac{3}{\phi(n)-2}\right)\left(1+\frac{1}{(\phi(n)-1)^{3}}\right) B_{\phi(n)-2} \quad(\bmod p)$,

where Euler function $\phi(n)=(p-1)(q-1)$, and we use Kummer congruence,

$$
\frac{B_{\phi(n)-2}}{\phi(n)-2} \equiv \frac{B_{p-3}}{p-3} \quad(\bmod p) .
$$

Similarly,

$$
Z(n) \equiv 6\left(1+\frac{3}{\phi(n)-2}\right)\left(1+\frac{1}{(\phi(n)-1)^{3}}\right) B_{\phi(n)-2} \quad(\bmod q),
$$

Therefore, by Chinese Remainder Theorem, we have

$$
Z(n) \equiv 6\left(1+\frac{3}{\phi(n)-2}\right)\left(1+\frac{1}{(\phi(n)-1)^{3}}\right) B_{\phi(n)-2} \quad(\bmod n) .
$$


Proof of Theorem 2. If $\alpha=1, \beta=1$, Theorem 2 is Theorem 1. Without loss of generality, suppose $\alpha \geq 2, \beta \geq 1$, similar to the proof of Theorem 1, we have

$$
\begin{aligned}
& Z\left(p^{\alpha} q^{\beta}\right)=\sum_{\substack{i+j+k=p^{\alpha} q^{\beta} \\
i, j, k \in \mathcal{P}_{p q}}} \frac{1}{i j k} \\
& =\sum_{\substack{i+j+k=p^{\alpha} q^{\beta} \\
i, j, k \in \mathcal{P}_{p}}} \frac{1}{i j k}-3 \sum_{\substack{a=1 \\
(a, p)=1}}^{p^{\alpha} q^{\beta-1}-1} \frac{1}{\left(p^{\alpha} q^{\beta}-a\right) q} \sum_{\substack{i+j=a q \\
(i j, p)=1}} \frac{1}{i j}+2 \sum_{\substack{a+b+c=p^{\alpha} q^{\beta-1} \\
a, b, c \in \mathcal{P}_{p}}} \frac{1}{a q b q c q} \\
& \equiv \sum_{\substack{i+j+k=p^{\alpha} q^{\beta} \\
i, j, k \in \mathcal{P}_{p}}} \frac{1}{i j k}+\frac{2}{q^{3}} \sum_{\substack{a+b+c=p^{\alpha} q^{\beta-1} \\
a, b, c \in \mathcal{P}_{p}}} \frac{1}{a b c}+\frac{6}{q^{2}} \sum_{\substack{a=1 \\
(a, p)=1}}^{p^{\alpha} q^{\beta-1}-1} \frac{1}{a^{2}} \sum_{\substack{i=1 \\
(i, p)=1}}^{a q-1} \frac{1}{i}
\end{aligned}
$$

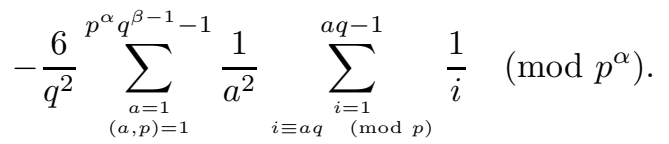

By Lemma 1, Lemma 5 and Lemma 8, (10) is congruent to

$$
\begin{aligned}
& \left(q^{\beta}+\frac{2 q^{\beta-1}}{q^{3}}\right)\left(-2 p^{\alpha-1}\right) B_{p-3}+\frac{6}{q^{2}} p^{\alpha-1} q^{\beta-1} q^{2} B_{p-3}-\frac{6}{q^{2}} \frac{q^{3}-q}{3 q} p^{\alpha-1} q^{\beta-1} B_{p-3} \\
\equiv & 2(2-q)\left(1-\frac{1}{q^{3}}\right) p^{\alpha-1} q^{\beta-1} B_{p-3} \quad\left(\bmod p^{\alpha}\right) .
\end{aligned}
$$

This completes the proof of Theorem 2 .

Proof of Theorem 3. By Theorem 2, if and only if one of the following cases is true, $Z\left(p^{\alpha} q^{\beta}\right) \equiv 0\left(\bmod p^{\alpha} q^{\beta}\right)$.
(a) $\begin{cases}p \equiv 2 & (\bmod q) \\ q \equiv 2 & (\bmod p)\end{cases}$
(b) $\left\{\begin{array}{l}p \equiv 2 \quad(\bmod q) \\ q^{3} \equiv 1 \quad(\bmod p)\end{array}\right.$
(c) $\begin{cases}p^{3} \equiv 1 \quad(\bmod q) \\ q \equiv 2 \quad(\bmod p)\end{cases}$
or $(d)\left\{\begin{array}{lll}p^{3} & \equiv 1 \quad(\bmod q) \\ q^{3} & \equiv 1 \quad(\bmod p)\end{array}\right.$

It is obvious that there are no primes $p, q$ satisfying Case $(a)$.

For Case $(b)$. Let $p=2+a q$, with $a$ odd. If $a=1, q^{3} \equiv-8 \equiv 1(\bmod p)$, then $p=3, q=1, q$ is not a prime. Hence $a \geq 3$. Since $q^{3} \equiv 1(\bmod p)$, then $q \equiv 1(\bmod p)$ or $q^{2}+q+1 \equiv 0(\bmod p)$. It is obvious that there are no primes $p, q$ satisfy $p \equiv 2(\bmod q)$ and $q \equiv 1(\bmod p)$. If $q^{2}+q+1 \equiv 0$ $(\bmod p)$, let $q^{2}+q+1=b p$, then $q^{2}+q+1=2 b+b a q$. Hence $q \mid 2 b-1$, there exists a positive integer $c$ such that $2 b-1=c q$. Therefore, $b p=\frac{(c q+1)(2+a q)}{2}=$ $\frac{a c}{2} q^{2}+\frac{a+2 c}{2} q+1>q^{2}+q+1$, impossible!

Similarly, we can show that Case $(c)$ is impossible too. 
For Case $(d)$. It is obvious that $p \equiv 1(\bmod q)$ and $q \equiv 1(\bmod p)$ are not possible. Similar to Case $(b)$, it can be justified that if $p \equiv 1(\bmod q)$ and $q^{2}+q+1 \equiv 0(\bmod p)$, then $q^{2}+q+1$ must be a prime. It is true if we exchange $p$ with $q$.

This completes the proof of Theorem 3 .

Remark 2 By Theorem 3, we know if and only if prime pairs $(p, q)$ satisfy $q=p^{2}+p+1$ or $p=q^{2}+q+1$ or $p \mid q^{2}+q+1$ and $q \mid p^{2}+p+1$, then $Z\left(p^{\alpha} q^{\beta}\right) \equiv 0$ $\left(\bmod p^{\alpha} q^{\beta}\right)$. There exist prime pairs $(p, q)$ such that $q=p^{2}+p+1$, for example $(p, q)=(3,13),(5,31),(17,307),(41,1723),(59,3541),(71,5113),(89,8011)$, etc..

Problem 1 Are there infinitely many prime pairs $(p, q)$ such that $q=$ $p^{2}+p+1 ?$

In 2004, Chao 1 proposed that: Find all pairs of positive integers $a$ and $b$ such that $a$ divides $b^{2}+b+1$ and $b$ divides $a^{2}+a+1$. There recurrence is: $a(1)=a(2)=1$ and $a(n+1)=\frac{1+a(n)+a^{2}(n)}{a(n-1)}$ for $n>2$, then

$$
a(n)=\left(\frac{4}{3}-\frac{2 \sqrt{21}}{7}\right)\left(\frac{5+\sqrt{21}}{2}\right)^{n}+\left(\frac{4}{3}+\frac{2 \sqrt{21}}{7}\right)\left(\frac{5-\sqrt{21}}{2}\right)^{n}+\frac{1}{3} .
$$

In fact, the pairs $(a(n), a(n+1)), n>0$, are all the solutions [2]:

$a(n)$ for $n=1, \cdots, 28$ are: 1, 1, 3, 13, 61, 291, 1393, 6673, 31971, 153181, 733933, 3516483, 16848481, 80725921, 386781123, 1853179693, 8879117341, 42542407011, 203832917713, 976622181553, 4679277990051, 22419767768701, 107419560853453, 514678036498563, 2465970621639361, 11815175071698241, $56609904736851843,271234348612560973$.

We find three prime pairs $(p, q)$ such that $p \mid q^{2}+q+1$ and $q \mid p^{2}+p+1$, i.e., $(p, q)=(3,13),(13,61),(22419767768701,107419560853453)$. What is the next pair?.

Problem 2 Are there infinitely many prime pairs $(p, q)$ such that $p \mid q^{2}+q+1$ and $q \mid p^{2}+p+1$ ?

Remark 3 By the conjecture and Chinese Remainder Theorem, we can count out the remainder of $Z(n)$ modulo $n$ for any positive integer $n$. However, we still have the problem as pointed out in [7, i.e.

Problem 3 Can we find an arithmetical function $f(n)$ such that

$$
Z(n) \equiv f(n) \quad(\bmod n) .
$$

Acknowledgements The authors wish to thank Dr. Deyi Chen for his kind help in calculation, especially in verifying the conjecture. 


\section{References}

[1] W. W. Chao, Problem 2981, Crux Mathematicorum, 30 (2004):430.

[2] https://oeis.org/A101368.

[3] C. G. Ji, A simple proof of a curious congruence by Zhao, Proc. Amer. Math. Soc., 133(2005): 3469-3472.

[4] R. Meštrović, Wolstenholme's theorem: its generalizations and extensions in the last hundred and fifty years(1862-2012), arXiv:1111.3057v2.

[5] $\breve{S}$ tefan Porubsky, Further congruences invoving Bernoulli numbers, J. Number Theory 16(1983): 87-94.

[6] I. S. Slavutskii, Leudesdorfs theorem and Bernoulli numbers, Arch. Math. (Brno) 35 (1999): 299-303.

[7] L. Q. Wang, T. X. Cai, A curious congruence modulo prime powers, J. Number Theory 144(2014): 15-24.

[8] B. Z. Xia, T. X. Cai , Bernoulli numbers and congruences for harmonic sums, Int. J. Number Theory, 6(2010): 849-855.

[9] J. Q. Zhao, A super congruence involving multiple harmonic sums, arXiv:1404.3549.

[10] J. Q. Zhao, Bernoulli Numbers, Wolstenholmes Theorem, and $p^{5}$ Variations of Lucas Theorem, J. Number Theory 123(2007): 18-26.

[11] X. Zhou, T. X. Cai , A Generalization of a Curious Congruence on Harmonic Sums. Proc. Amer. Math. Soc., 135 (2007): 1329-1333. 